\section{Hydrazide Derivatives of Luminol for Chemiluminescence-Labelling of Macromolecules}

\author{
Takayuki Shibata, ${ }^{*, a, b}$ Hiroki Yoshimura, ${ }^{b}$ \\ Asako Yamayoshi, ${ }^{b}$ Nobuaki Tsuda, ${ }^{c}$ and \\ Shpend Dragusha ${ }^{d}$
}

${ }^{a}$ Department of Laboratory Sciences, Gunma University Graduate School of Health Sciences; 3-39-22 Showa-machi, Maebashi, Gunma 371-8514, Japan: ${ }^{b}$ Graduate School of Biomedical Sciences, Nagasaki University; 1-14 Bunkyo-machi, Nagasaki, Nagasaki 852-8521, Japan: ' Center for Industry, University and Government Cooperation, Nagasaki University; 1-14, Bunkyo-machi, Nagasaki, Nagasaki 852-8521, Japan: and ${ }^{d}$ Faculty of Pharmacy, UBT-Higher Education Institution; Lagjja Kalabria p.n., 10000 Prishtina, Kosovo.

Received February 13, 2019; accepted May 9, 2019; advance publication released online May 17, 2019

A series of chemiluminescent compounds containing a hydrazide group as a nucleophilic functional group has been synthesized. The syntheses were started from chemiluminescent luminol and isoluminol. The linker moiety was easily introduced onto non-nucleophilic exocyclic amino groups of luminol and isoluminol by gentle heating with cyclic acid anhydrides such as glutaric anhydride. The resulting carboxy group was converted to hydrazide by a simple condensation reaction using carbodiimide. Although majority of the synthesized compounds did not emit strong light, a sufficient chemiluminescence intensity was obtained from luminolamido-C2-hydrazide ( $\mathrm{L2H}$ ) comprising of luminol scaffold with a dimethylene linker. The ability of $\mathrm{L} 2 \mathrm{H}$ to form a covalent bond with a macromolecule was further investigated by incubation with oxidized horseradish peroxidase. The analysis on matrix assisted laser desorption/ionization-time of flight (MALDI-TOF) MS revealed that the coupling efficiency of L2H was similar to that of commercially available labelling reagent having a hydrazide group. These results suggested that $\mathrm{L2H}$, the luminol hydrazide containing a dimethylene linker, could be useful for the labelling of macromolecules in the sensitive bioassay such as chemiluminescence immunoassay.

Key words luminol; chemiluminescence; labelling reagent; hydrazide

\section{Introduction}

Introduction of luminous molecules into biomolecules is a fundamental experimental technique for bioanalyses such as immunoassay and DNA hybridization assay. Especially, chemiluminescent (CL)-labelling has become a common method due to the beneficial wide dynamic range and high sensitivity of the CL detection. ${ }^{1)}$ A typical CL detection system utilizes CL-active enzymes i.e., peroxidase, ${ }^{2)}$ phosphatase $^{3)}$ and $\beta$-galactosidase. ${ }^{4)}$ These enzymes are covalently tethered with detection probes such as antibodies and oligonucleotides for specific recognition of target molecules. ${ }^{5}$ Biotinfunctionalized enzymes have also been used for the detection of biotinylated target-specific probes through biotin-avidin interaction. ${ }^{5)}$ However, instability of the enzymes at ambient temperature might lead to reduced reproducibility, ${ }^{6)}$ and elongation of reaction time significantly increases the background signal. Therefore, a non-enzymatic CL-labelling small molecule could provide a powerful tool for the accurate and sensitive bioanalysis.

In contrast to widely-used fluorescence-labelling reagents, there are only limited number of CL-labelling small molecules available. One of the possible reasons why CL-labelling agents have not been developed is that the CL substance is very sensitive to a chemical modification which might cause a decrease of its CL intensity. In case of luminol, a common CL substance, an exocyclic amino group has low nucleophilicity and pristine luminol has not been used for the direct labelling of macromolecules. Therefore, a linker should be introduced to the phthalazine moiety. The convenient way to attach a linker is the introduction of an acyl group or an alkyl group onto the 5-amino group. However, such modifications have been known to diminish the $\mathrm{CL}$ intensity. ${ }^{7,8)}$ Isoluminol is an alternative CL substrate, and several isoluminol derivatives having reactive functional groups have been developed. ${ }^{9-12)}$ Although these isoluminol derivatives represented sufficient reactivity to electrophilic functional groups, the CL intensities obtained from them are limited due to the less luminosity of isoluminol than luminol.

\section{Results}

Acylation of the exocyclic amino groups of luminol/isoluminol has been commonly conducted using acid chlorides which is highly reactive and requires a strict anhydrous condition. We found that succinic anhydride reacts with luminol with gentle heating to give the corresponding acylated product with a terminal carboxy group (Fig. 1). Luminol is hardly soluble in acidic to neutral water and dissolves well in alkaline water. The product of this reaction showed similar solubility to luminol, and the pure product was obtained in a good yield by acidification of the reaction mixture (see NMR spectrum, Supplementary Materials). Hydrazide is a well-known nucleophile derived from a carboxy group. The resulting terminal carboxyl group was converted to a hydrazide group using hydrazine monohydrate and 1-ethyl-3-(3dimethylaminopropyl)-carbodiimide (EDC) in the presence of 1-hydroxy-1H-benzotriazole (HOBt) (Fig. 1). The hydrazide form, namely luminol-amido-C2-hydrazide (L2H), was also obtained in a good yield by the same purification procedure as the carboxylic acid form. Therefore, we synthesized a series of derivatives using luminol and isoluminol as CL backbones, and succinic acid and glutalic acid as linker moieties (Fig. 1). The same precipitation method was applied to the purification of the first step products, of which yields were similar (Table 1). However, the products derived from glutalic anhydride ( $\mathbf{L 3 H}$ and $\mathbf{~ 3 3 H}$ ) gave less precipitation at the 2nd purification stage. Although the pure products were isolated by changing the purification method (see Methods, Supplementary Materials), the yields for both $\mathbf{L} \mathbf{3 H}$ and $\mathbf{I 3 H}$ were only moderate.

The CL intensities of the synthesized luminol derivatives were next evaluated using hydrogen peroxide under basic con- 
(A)<smiles>CC1CC(=O)OC(=O)C1C(=O)O</smiles><smiles>NC(=O)[C@@H](N)C(=O)O</smiles><smiles>NNC(=O)C(=O)Nc1cccc2c(=O)[nH][nH]c(=O)c12</smiles>

(B)

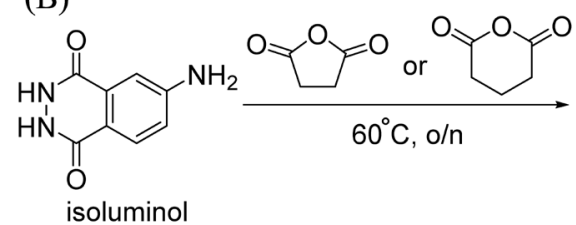

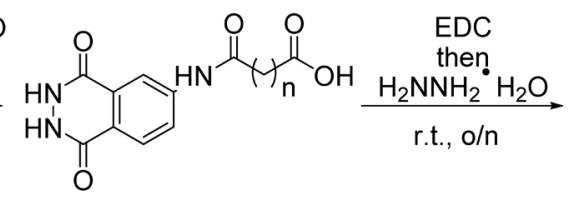

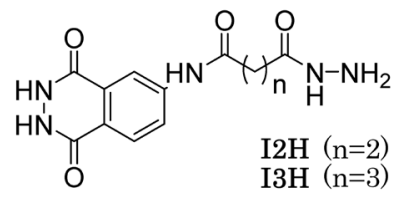

Fig. 1. Synthetic Route to (A) Luminol and (B) Isoluminol Derivatives Having a Hydrazide Group

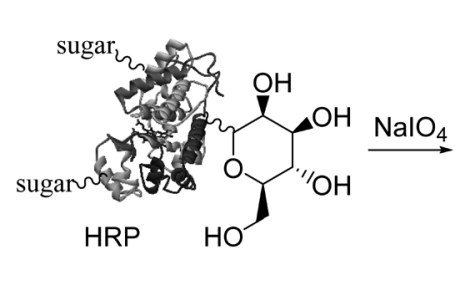

Formation of multiple aldehyde groups

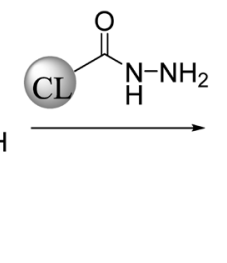

Introduction of multiple

CL substances

Fig. 2. Schematic Illustration of the Incorporation of the Synthesized Hydrazide Compounds into HRP

Table 1. The Yields of Acylation and Hydrazidation Reactions

\begin{tabular}{ccccc}
\hline \hline Entry & Backbone & $n$ & Yield $1^{a)}(\%)$ & Yield $2^{b)}(\%)$ \\
\hline 1 & Luminol & 2 & 92.0 & 78.9 \\
2 & & 3 & 90.0 & 35.8 \\
3 & Isoluminol & 2 & 80.8 & 82.9 \\
4 & & 3 & 59.7 & 44.0 \\
\hline
\end{tabular}

a) The isolated yield from the condensation reaction with acid anhydrides. $b$ ) The isolated yield from the hydrazidation reaction.

Table 2. CL Properties of the Synthesized Molecules

\begin{tabular}{ccc}
\hline \hline Entry & Compound & $\left.\mathrm{RCI}^{a}\right)\left(\times 10^{6}\right.$ count $)$ \\
\hline 1 & L2H & $1.93 \pm 0.60$ \\
2 & L3H & $0.29 \pm 0.04$ \\
3 & I2H & $<0.01$ \\
4 & I3H & $<0.01$ \\
5 & Luminol & $6.50 \pm 0.24$ \\
6 & Isoluminol & $0.72 \pm 0.15$ \\
\hline
\end{tabular}

a) Relative CL intensity \pm standard deviation (S.D.) accumulated for $10 \mathrm{~min}$ using $0.5 \mathrm{mM}$ of each compound $(n=3)$.

dition. $\left.{ }^{6}\right)$ The relative CL intensity (RCI) of luminol was approximately 10 times higher than isoluminol (Table 2), which is consistent of the reported data. ${ }^{13)}$ The RCI of $\mathbf{L 2 H}$ was 3.4 times lower than that of luminol and 2.6 times higher than that of isoluminol. It has been reported that the RCI obtained by isoluminol derivatives did not exceed the RCI of isoluminol, ${ }^{14)}$ indicating that $\mathbf{L} \mathbf{2 H}$ emitted relatively strong CL. $\mathbf{L} \mathbf{3 H}$, having just one carbon longer linker than $\mathbf{L} \mathbf{2 H}$, exhibited very weak light which was 2.5 times weaker than isoluminol. As mentioned above, chemical modification of the CL substance often results in quenching of its CL intensity. This might be due to the difference of the distance between the amino group of the hydrazide and the oxygen of the phthalazinedione ring. ${ }^{15)}$ Both isoluminol derivatives, $\mathbf{I 2 H}$ and $\mathbf{I 3 H}$, showed negligible strength of light. These results suggested that $\mathbf{L} \mathbf{2 H}$ has a potential as the reagent for the direct CL labelling of macromolecules.

Hydrazide has been utilized for introducing small molecules into bioactive materials for biological/biochemical assays. For example, biotin-avidin interaction is a widespread use in bioassay and biotinylation of the target of interest can sometimes be achieved by using a modified biotin having a hydrazide group. ${ }^{16)}$ In this case, sugar moiety in the target molecule should be oxidized by periodate to build multiple aldehyde groups for the formation of Schiff base. Thus the labelling efficiency of $\mathbf{L} \mathbf{2 H}$ was investigated using horseradish peroxidase (HRP) as a model target molecule. HRP is a glycoprotein with the molecular weight of $\left.44 \mathrm{kDa}{ }^{17}\right)$ The sugar moiety of HRP can be oxidized with peroxidase to produce aldehyde groups which react with hydrazide compounds to form Schiff bases $^{18)}$ (Fig. 2). An aqueous solution of HRP was treated with sodium periodate, followed by incubation with $10 \mathrm{eq}$ of the labeling reagents dissolved in dimethyl sulfoxide. The resulting mixtures were directly applied to matrix assisted laser desorption/ionization-time of flight (MALDI-TOF) MS to calculate the amount of labeling reagents tethered with HRP (Table 3). The average number of $\mathbf{L 2 H}$ incorporated was 4.1 molecules per one molecule of HRP. This result indicated that L2H has the ability to react to aldehyde groups and multiple number of $\mathbf{L 2 H}$ can be introduced into a target molecule. The 
Table 3. Labeling Efficiency to Oxidized HRP

\begin{tabular}{|c|c|c|c|c|c|}
\hline Reagents & & $1 \mathrm{st}$ & 2 nd & $3 \mathrm{rd}$ & Average number \\
\hline \multirow[t]{3}{*}{ L2H (MW = 277.2) } & Found & 44545.3 & 44183.3 & 44183.4 & 4.1 \\
\hline & $\Delta^{a)}$ & 1290.3 & 928.3 & 928.4 & \\
\hline & Number $^{b)}$ & 5.0 & 3.6 & 3.6 & \\
\hline \multirow{3}{*}{$\begin{array}{l}\text { Biotin AC5 hydrazide } \\
\quad(\mathrm{MW}=371.5)\end{array}$} & Found & 46365.2 & 44680.5 & 44930.4 & 5.8 \\
\hline & $\Delta$ & 3110.2 & 1425.5 & 1675.4 & \\
\hline & Number & 8.8 & 4.0 & 4.7 & \\
\hline \multirow[t]{3}{*}{ Luminol $(\mathrm{MW}=177.2)$} & Found & 43238.2 & 43219 & - & Approx. 0 \\
\hline & $\Delta$ & -16.8 & -35.9 & - & \\
\hline & Number & $(-0.1)$ & $(-0.2)$ & - & \\
\hline
\end{tabular}

a) Increased molecular mass from molecular weight of HRP $\left.(43255) \cdot{ }^{17)} b\right)$ Calculated by the following equation: number $=\Delta /(\mathrm{MW}-18)$.

reason why the coupling efficiency was not constant in three repeated syntheses would be because the oxidation and conjugation reactions were carried out without stirring in order to retaining the tertiary structure of the target macromolecule. The labelling efficiency was compared with a commercially available biotinylation reagent (biotin-AC5-hydrazide) which contains a long linker chain and the terminal hydrazide group. The average number of biotin incorporated was 5.8, which is a little more than $\mathbf{L} \mathbf{2 H}$. It should be noted that pristine luminol exhibited no conjugation to HRP. A rigid structure of luminol as well as the intermolecular hydrogen bonding might prevent the 5-amino group from reaching close proximity to the aldehyde groups in a bulky protein, and the hydrazide group attached to the flexible linker is beneficial for the labeling of macromolecules.

\section{Conclusion}

In conclusion, we synthesized a series of luminol derivatives having a hydrazide group by an easy derivatization method. Each reaction was simple and each purification was not laborious, and the reaction yields were satisfactory. Especially, L2H showed higher CL than isoluminol, suggesting that the quantum yield of $\mathbf{L 2 H}$ would be relatively higher in comparison with the other chemiluminescence-labelling small molecules developed so far. Multiple number of $\mathbf{L 2 H}$ was successfully introduced into the target macromolecule, and the labeling efficiency was comparable to the widely-used biotinylation reagent having a hydrazide group. It has been known that the CL intensity of the luminol-labeled protein increases as the number of luminol moiety in the protein increases ${ }^{19)}$ Since the introduction of multiple CL substances into a macromolecule allows the enrichment of CL signals, this technique has been applied to the sensitive detection of biomolecules such as proteins and nucleic acids. ${ }^{19,20)}$ We expect that $\mathbf{L 2 H}$ could be useful as a direct CL-labelling reagent in bioassays.

Acknowledgments This work was partly supported by Grants-in-Aid for Scientific Research from Japan Society for the Promotion of Science (JSPS), and partly supported by the research grant of Astellas Foundation for Research on Metabolic Disorders.

Conflict of Interest The authors declare no conflict of interest.

Supplementary Materials The online version of this article contains supplementary materials.

\section{References}

1) Roda A., Mirasoli M., Michelini E., Di Fusco M., Zangheri M., Cevenini L., Roda B., Simoni P., Biosens. Bioelectron., 76, 164-179 (2016).

2) Zhang Z., Lai J., Wu K., Huang X., Guo S., Zhang L., Liu J., Talanta, 180, 260-270 (2018).

3) Shan S., He Z., Mao S., Jie M., Yi L., Lin J. M., Talanta, 171, 197-203 (2017).

4) Yang X., Janatova J., Andrade J. D., Anal. Biochem., 336, 102-107 (2005).

5) Fan A., Cao Z., Li H., Kai M., Lu J., Anal. Sci., 25, 587-597 (2009). 6) Lu J., Lau C., Morizono M., Ohta K., Kai M., Anal. Chem., 73, 5979-5983 (2001).

7) Omote Y., Miyake T., Ohmori S., Sugiyama N., Bull. Chem. Soc. Jpn., 39, 932-935 (1966).

8) Omote Y., Miyake T., Ohmori S., Sugiyama N., Bull. Chem. Soc. Jpn., 40, 899-903 (1967)

9) Xie S., Dong Y., Yuan Y., Chai Y., Yuan R., Anal. Chem., 88, 5218-5224 (2016).

10) Calvo-Muñoz M. L., Dupont-Filliard A., Billon M., Guillerez S. Bidan G., Marquette C., Blum L., Bioelectrochemistry, 66, 139-143 (2005).

11) Palmioli A., Crisma M., Peggion C., Brusasca P., Zanin D., Dal Corso A., Ingallinella P., Peri F., Tetrahedron Lett., 54, 4446-4450 (2013).

12) Pazzagli M., Messeri G., Caldini A. L., Moneti G., Martinazzo G., Serio M., J. Steroid Biochem., 19 (1B), 407-412 (1983).

13) Rájecký M., Lojek A., Cíž M., Int. J. Lab. Hematol., 34, 136-142 (2012).

14) Schroeder H. R., Yeager F. M., Anal. Chem., 50, 1114-1120 (1978).

15) Jancinová V., Drábiková K., Nosál R., Racková L., Májeková M., Holománová D., Redox Rep., 11, 110-116 (2006).

16) Bayer E. A., Ben-Hur H., Wilchek M., Anal. Biochem., 170, $271-$ 281 (1988).

17) Welinder K. G., Eur. J. Biochem., 96, 483-502 (1979).

18) Morehead H. W., Talmadge K. W., O'Shannessy D. J., Siebert C. J., J. Chromatogr. A, 587, 171-176 (1991).

19) Messeri G., Schroeder H. R., Caldini A. L., Orlando C., Methods Enzymol., 133, 557-568 (1986).

20) Chai Y., Tian D., Cui H., Anal. Chim. Acta, 715, 86-92 (2012). 